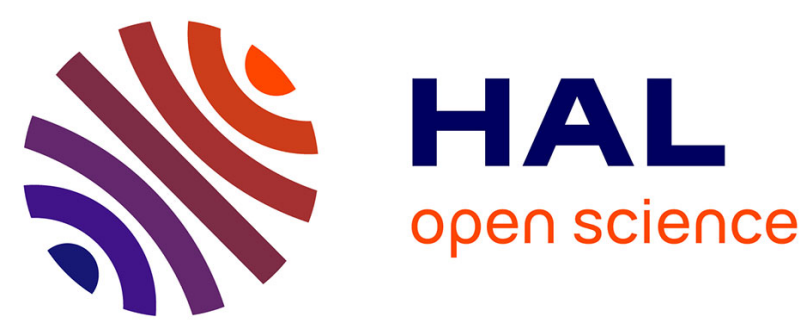

\title{
The Sustainability of Quinoa Production in Southern Bolivia: from Misrepresentations to questionable solutions. Comments on S.Jacobsen (2011, J. Agron. Crop Sci.36197:390-399)
}

Thierry Winkel, H D Bertero, Pierre Bommel, Jean Bourliaud, M Chevarría Lazo, Genevieve Cortes, Pierre Gasselin, Sam Geerts, Richard Joffre, François Léger, et al.

\section{- To cite this version:}

Thierry Winkel, H D Bertero, Pierre Bommel, Jean Bourliaud, M Chevarría Lazo, et al.. The Sustainability of Quinoa Production in Southern Bolivia: from Misrepresentations to questionable solutions. Comments on S.Jacobsen (2011, J. Agron. Crop Sci.36197:390-399). Journal of Agronomy and Crop Science, 2012, 198 (4), p. 314-319. 10.1111/j.1439-037X.2012.00506.x . ird-02381033

HAL Id: ird-02381033 https://hal.ird.fr/ird-02381033

Submitted on 27 Nov 2019

HAL is a multi-disciplinary open access archive for the deposit and dissemination of scientific research documents, whether they are published or not. The documents may come from teaching and research institutions in France or abroad, or from public or private research centers.
L'archive ouverte pluridisciplinaire HAL, est destinée au dépôt et à la diffusion de documents scientifiques de niveau recherche, publiés ou non, émanant des établissements d'enseignement et de recherche français ou étrangers, des laboratoires publics ou privés. 


\section{La durabilité de la production de quinoa au sud de la Bolivie :}

2

Jacobsen (2011, J. Agron. Crop Sci. 197(5): 390-399)

\section{Auteurs}

Thierry WINKEL (1), H. Daniel BERTERO (2), Pierre BOMMEL (3), Jean BOURLIAUD (4), Marco CHEVARRÍA LAZO (4), Geneviève CORTES (5), Pierre GASSELIN (6), Sam GEERTS (7), Richard JOFFRE (8), François LÉGER (9), Brigido MARTINEZ AVISA (10), Serge RAMBAL (8), Gilles RIVIÈRE (11), Muriel TICHIT (9), Jean-François TOURRAND (3), Anaïs VASSAS TORAL (5), Jean-Joinville VACHER (1), Manuela VIEIRA PAK (3)

1. IRD, CEFE-CNRS, F-34293 Montpellier cedex 5, France

2. Universidad de Buenos Aires, Facultad de Agronomía, Cátedra de Producción Vegetal, C1417DSE, Buenos Aires, Argentine

3. CIRAD, UR GREEN, F-34398 Montpellier cedex 5, France

4. INRA, UR MONA, F-94205 Ivry-sur-Seine, France

5. Université Paul Valéry, UMR 5281 ART-Dev, F-34199 Montpellier cedex 5, France

6. INRA, UMR INNOVATION, F-34060 Montpellier cedex 1, France

7. Katholieke Universiteit, Department de Earth et Environnemental Sciences, B-3001 Leuven, Belgique

8. CNRS, UMR 5175 CEFE, F-34293 Montpellier cedex 5, France

9. INRA, UMR SAD-APT, F-75231 Paris cedex 5, France

10.ANAPQUI, Asociación Nacional de Productores de Quinua, La Paz, Bolivie

11.EHESS, CERMA, UMR 8168 MASCIPO, F-75013 Paris, France

\section{Contacts}

Thierry WINKEL

CEFE-CNRS/IRD

F-34293 Montpellier cedex 5, France

Tel: 0467613255 - Email: thierry.winkel@ird.fr - Web: www.ird.fr/equeco

\section{Citation de l'article original}

Winkel T, Bertero HD, Bommel P, Bourliaud J, Chevarría Lazo M, Cortes G, Gasselin P, Geerts S, Joffre R, Léger F, Martinez Avisa B, Rambal S, Rivière G, Tichit M, Tourrand J-F, Vassas Toral A, Vacher J-J, Vieira Pak M. 2012. The sustainability of quinoa production in southern Bolivia: from misrepresentations to questionable solutions. Comments on S. Jacobsen (2011, J. Agron. Crop Sci. 197: 390-399). Journal of Agronomy and Crop Science, 198: 314-319. 


\section{Résumé}

Analysant la situation de la production de quinoa dans le sud de la Bolivie, premier exportateur mondial, Jacobsen (2011, J. Agron. Crop Sci. 197 : 390) affirme que le boom du marché d'exportation a des effets négatifs sur le milieu naturel et sur la consommation locale de quinoa, ce qui conduit à un désastre écologique dans la région. Considérant le peu d'information scientifique sur les rapides changements sociaux et environnementaux en cours dans la région, nous jugeons erronée l'analyse de la situation de la production de quinoa dans l'altiplano sud de la Bolivie présentée par Jacobsen. Nous soutenons notamment que : i) les données présentées par Jacobsen (2011) ne démontrent aucunement une chute des rendements de quinoa supposée refléter une dégradation des sols, ii) l'argumentaire de Jacobsen (2011) concernant la consommation locale de quinoa est infondé tant du point de vue nutritionnel que socio-culturel. Nous estimons que la diffusion des arguments développés par Jacobsen (2011), en dépit ou à cause de leurs lacunes, peut avoir un impact très négatif sur les personnes préoccupées par une production alimentaire durable et un commerce équitable avec les pays en développement. Nous concluons que, plutôt que de contrôles agrotechniques renforcés sur les producteurs locaux, la concurrence croissante sur le marché international de la quinoa a besoin d'ajustements vers une économie et une recherche en coopération plus éthiques.

\section{Mots-clés}

Andes; Bolivie; agriculture durable; consommation alimentaire; dégradation des sols; éthique scientifique; quinoa

60

\section{Abstract}

Reviewing the situation of quinoa production in southern Bolivia, Jacobsen (2011, J. Agron. Crop Sci. 197: 390) argues that the booming export market has a negative effect on the environment and on the home consumption of quinoa, thereby leading to an environmental disaster in the region. In view of the scarcity of scientific knowledge on the rapid social and environmental dynamics in the region, we consider that Jacobsen's review misrepresents the situation of quinoa production in southern Bolivia. Specifically, we argue that: i) the data presented by Jacobsen (2011) does not support any drop in quinoa crop yield supposed to reflect soil degradation, ii) his demonstration regarding home consumption of quinoa is ill-founded from both a nutritional and a cultural point of view. We suggest that the diffusion of the arguments exposed by Jacobsen (2011), because of their flaws, might have strong negative impacts on those concerned with sustainable food production and fair-trade with developing countries. We conclude that, rather than reinforced agro-technical controls on local farmers, the rising competition in the international quinoa market requires a shift towards an ethical economy and ethical research cooperation with quinoa producers.

\section{Keywords}

Andes; Bolivia; Chenopodium quinoa; food consumption; soil degradation; scientific ethics; sustainable agriculture; 


\section{Introduction}

Un article récent de Jacobsen (2011) examine la situation de la production de quinoa dans le sud de la Bolivie considérant les changements environnementaux et socioéconomiques qu'a connu cette production originaire des Andes depuis son entrée, à la fin des années 80 , dans un marché d'exportation florissant. Au vu des changements radicaux intervenus dans la production de quinoa, l'auteur affirme que "le développement d'un marché d'exportation peut avoir un effet négatif sur l'environnement et sur la consommation locale de ce produit", ce qui conduirait à un désastre écologique dans la région. Selon l'analyse de Jacobsen (2011), la production de quinoa a donc un besoin urgent d'ajustements techniques, tels que des systèmes d'irrigation sophistiqués et des contrôles renforcés sur la production locale de quinoa.

Malgré un intérêt croissant dans le commerce mondial, la littérature scientifique sur la quinoa reste rare comparée à celle d'autres cultures alimentaires, d'autant plus si l'on s'intéresse à une zone de production particulière comme l'altiplano sud de Bolivie, premier exportateur mondial de ce produit. Rapportant les conclusions d'un atelier international sur la durabilité de la production végétale dans cette région, Reynolds et al. (2008a: 11) soulignent que très peu de données sont disponibles sur les changements d'érosion des sols dans différents territoires, et encore moins sur les facteurs critiques pour la durabilité de la production végétale dans la région. Considérant ce manque d'études scientifiques d'une part, et la rapidité des changements sociaux et environnementaux dans la région de l'autre, nous considérons que l'analyse de Jacobsen sur la situation de la production de quinoa dans le sud de la Bolivie est hâtive et erronée. Nous montrons ici que :

1. une ré-analyse des données présentées par Jacobsen (2011) ne corrobore en aucune façon la chute des rendements de quinoa supposée refléter une dégradation du milieu pendant la période où la production s'est accrue ;

2. l'argumentaire développé Jacobsen (2011) au sujet de la consommation locale de quinoa est infondé tant du point de vue nutritionnel que socio-culturel.

Après avoir signalé quelques autres erreurs dans le diagnostic et les solutions présentés par Jacobsen (2011), nous suggérons que la diffusion de ces arguments, en dépit ou à cause de leurs lacunes, peut avoir un impact très négatif sur les personnes préoccupées par une production alimentaire durable et un commerce équitable avec les pays en développement.

\section{Les preuves d'un désastre environnemental ?}

La dégradation des sols due à l'usage intensif des tracteurs et à un accès restreint à la fumure animale sont les principaux problèmes environnementaux signalés par Jacobsen (2011: 392, 393). Pour étayer l'idée qu'une "sévère dégradation de la fertilité du sol" a eu lieu suite à l'expansion de la culture de quinoa, Jacobsen cite des publications du PIEB (2009) et d'ASPA II (2008). Mais le premier de ces documents n'est qu'un simple appel à projets de recherche, et le second un rapport interne en vue d'obtenir des financements, aucun des deux n'apportant de preuve scientifique d'une dégradation des sols dans la région. À notre avis, les conclusions tirées de ces publications par Jacobsen (2011) dépassent les connaissances actuelles sur la fertilité des sols dans l'altiplano sud de Bolivie, sans parler des facteurs qui lui seraient sous-jacents (durée de la jachère, dosage de la 
fertilisation organique ou utilisation des tracteurs). Néanmoins, se référant à une figure montrant le rendement de la quinoa en Bolivie sur la période 1961-2009, Jacobsen (2011: 391) affirme que : "avec l'accroissement des surfaces cultivées pendant les 10 dernières années, les rendements sont passés de près de $700 \mathrm{~kg} / \mathrm{ha}$ à $570 \mathrm{~kg} / \mathrm{ha}$ in 2009". Il suggère alors un lien direct entre une durée de jachère réduite et "la réduction progressive des rendements de quinoa sur les derniers 20 ans", citant Félix (2008). Hormis le fait que le rapport de projet de développement de Félix (2008) n'avait pas pour objectif une démonstration scientifique d'une relation de cause-à-effet entre durée de jachère, fertilité du sol et rendement en grain, la série de données de rendement de quinoa présentée par Jacobsen (2011) dans sa Figure 1 n'étaye visiblement pas son affirmation d'une baisse de rendement sur la période du boom de la quinoa. Au contraire, une ré-analyse de cette série de données (notre Fig. 1) montre que la pente de la régression temporelle n'est pas différente de zéro sur la période 1961-2009, et qu'elle augmente même significativement sur les dernières 20 années, contredisant l'affirmation de Jacobsen. En réalité, cette série de données de rendements de quinoa voit alterner deux périodes d'accroissement (1961-1975 et 1991-1997) avec deux périodes de réduction des rendements (1976-1990 et 1998-2009). De telles fluctuations interannuelles de rendement sont difficiles à interpréter en absence d'informations complémentaires sur les tendances climatiques, les changements du paysage, les pratiques de culture et la fertilité du sol dans la région (sans parler d'éventuels changements dans les méthodes utilisées par les instituts de statistiques...). De fait, avec un coefficient de variation de $20 \%$ sur la période 1961-2009, la production nationale de quinoa reste dans la gamme normale de variabilité interannuelle des rendements pour une culture produite avec peu d'intrants dans un milieu aride, et ne montre aucune tendance décroissante susceptible d'indiquer un "désastre écologique".

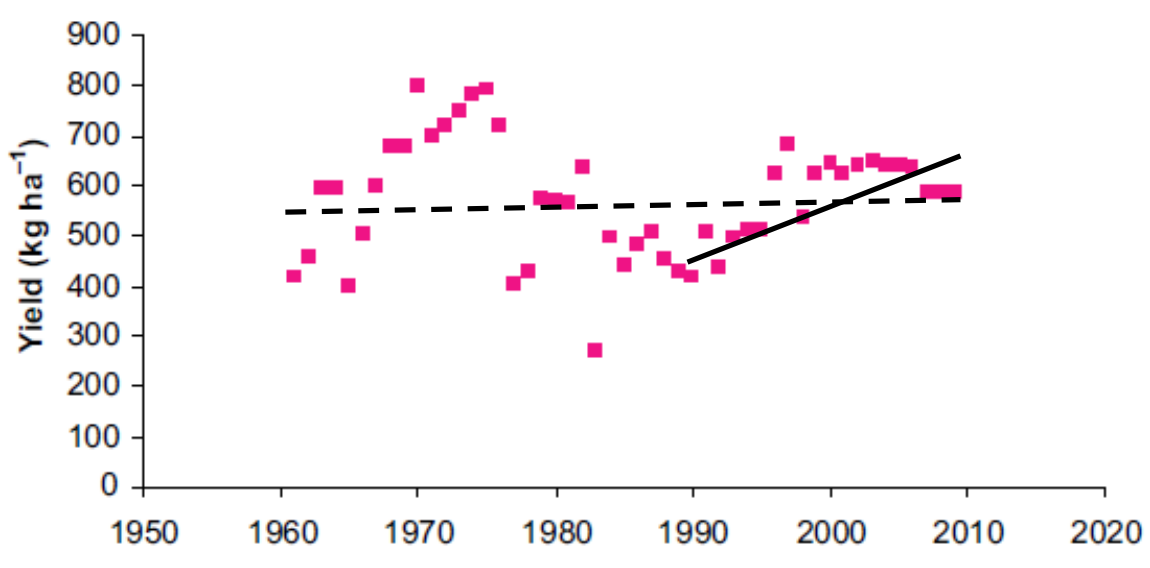

Fig. 1 Régressions statistiques sur la série de données de rendements de quinoa publiée par Jacobsen (2011). Période 1961-2009 (ligne pointillée): pente $=0.112 \pm 1.18, P=0.92$. Période 1990-2009 (ligne continue): pente $=8.64 \pm 2.18, P=0.0009$.

Propageant une erreur couramment faite par d'autres auteurs qu'il cite (Cossio 2008, Félix 2008), Jacobsen (2011) établit un lien direct entre une supposée tendance à la baisse des rendements annuels et un processus de dégradation du sol, oubliant que, du fait de l'interférence de nombreux 
phénomènes (attaques parasitaires, stress climatiques, pratiques de culture, etc.), une variable annuelle comme le rendement en grains ne permet pas de caractériser avec certitude un processus lent comme la dégradation des terres (Reynolds et al. 2007, 2008b). De plus, des statistiques nationales brutes, avec toutes les limites dont elles sont entachées dans un pays en développement comme la Bolivie, peuvent difficilement fournir des indicateurs fiables d'une crise environnementale à un niveau régional ou local. Une première conclusion de cette ré-analyse de l'argumentation de Jacobsen serait donc que des études détaillées et scientifiquement valides manquent encore et seraient pourtant cruciales pour caractériser avec certitude les processus reliant la production de quinoa à la gestion des sols dans cette région soumise à des changements rapides d'usage des terres et des pratiques agricoles.

\section{La consommation locale de quinoa}

Abordant la question de la consommation locale de quinoa, Jacobsen (2011: 392) assure que : "I'exportation de quinoa a augmenté depuis 2001 alors que la consommation domestique a baissé", faisant référence à sa Figure 2 , elle aussi basée sur des statistiques nationales. Tout d'abord, il faut souligner qu'ajuster une régression polynomiale à une série temporelle ne comptant que 12 points, affectée de plus par une forte variabilité interannuelle, ne peut conduire à des résultats statistiquement robustes. Une série beaucoup plus longue serait nécessaire pour tirer une conclusion valide concernant un changement significatif des habitudes alimentaires des populations locales. Ensuite, on voit mal comment des données brutes collectées au niveau national pourraient refléter le régime alimentaire des producteurs de quinoa de l'altiplano sud de Bolivie. C'est le même problème que celui évoqué plus haut : extrapoler à une échelle locale des conclusions tirées de données nationales. Nonobstant, Jacobsen (2011: 396) affirme que "les paysans de l'altiplano sud, où la quinoa Real à gros grains est produite pour l'exportation, ne consomment plus leur propre quinoa, à cause de sa valeur élevée sur le marché", ajoutant : "Des données d'enquêtes auprès des ménages ont montré que, pendant la durée de l'enquête, la majorité des repas ne comportait pas du tout de quinoa, ce qui suggère que la quinoa serait en passe de devenir une culture marginale (underutilized) chez les producteurs de quinoa eux-mêmes". Or, aucune donnée d'étude anthropologique ou sociologique n'est apportée pour corroborer ce changement supposé dans les habitudes alimentaires locales. Des données récentes sur la consommation de quinoa dans les familles paysannes de I'altiplano sud de Bolivie existent pourtant. Laguna (2008: 130) évalue ainsi l'auto-consommation de quinoa par les paysans de cette région entre 12 à $14 \%$ de leur propre production. Plus récemment, dans une étude sur 275 ménages de l'altiplano sud, Astudillo (2007: 24) rapporte que $40 \%$ des repas préparés la veille de l'enquête comportaient de la quinoa, avec des chiffres encore plus élevés dans les familles vivant loin des grands axes de communication. Acosta-Alba (2007: 25) dans une enquête sur 36 familles du nord et de l'ouest du Salar de Uyuni note que tous les producteurs de quinoa conservent environ $10 \%$ de leur production totale pour l'auto-consommation et le stock de semences. Héran (2011) observe que, quel que soit le volume de leur production, les producteurs de quinoa de l'est de cette même région gardent toujours une quantité de quinoa de 40 à $200 \mathrm{~kg} / \mathrm{an}$ pour leur consommation familiale. Ofstehage (2010: 21) dans une étude ethnographique au sud du Salar de Uyuni (Los Lipez) affirme qu' "une quantité notable de quinoa reste dans la région d'Uyuni pour l'auto-consommation". Enfin, bien qu'elle soit centrée sur la consommation de quinoa dans les zones urbaines, l'étude de Montoya (2007: 22, 25, 27) confirme que la plupart de la quinoa produite dans l'altiplano bolivien est consommée par les familles de producteurs. 
Plus généralement, nous voudrions souligner ici une confusion courante chez ceux qui se préoccupent de la part de la quinoa dans le régime alimentaire des familles paysannes de la région. C'est un fait reconnu que, sur une base purement quantitative, la quinoa représente une fraction plus faible dans le régime alimentaire local que les pâtes ou le riz (Rojas et al. 2004). De nombreux nutritionnistes, considérant la haute teneur en protéines de la quinoa, jugent qu'une augmentation substantielle de la quinoa dans le régime alimentaire des populations locales serait très bénéfique à leur santé. Cependant, il faut être circonspect dans l'appréciation du rôle de la quinoa dans le régime alimentaire local : une idée commune est que la part de la quinoa devrait être équivalente à celle d'autre féculents comme les tubercules, les fèves, le maïs, l'orge, les pâtes, le pain ou le riz. Or, et bien que la quinoa soit parfois promue dans les marchés des pays du Nord comme le "riz des Incas", les populations andines ne l'ont jamais consommée comme une céréale de base, à la manière du riz en Asie ou du blé au Proche-Orient et en Europe. De fait, les populations locales considèrent la quinoa comme un aliment "lourd", et déconseillent même d'en manger en trop grande quantité pour le dîner (Johnsson 1986: 107). Traditionnellement, la quinoa est utilisée avant tout pour épaissir des soupes ou des boissons (lahua, pesqe) ou sous forme de petits biscuits (kispiña, mukuna), et plus rarement comme plat principal (phisara) (National Research Council 1989, Tapia et al. 2000). Par conséquent, il est incorrect de comparer sur une base quantitative les parts respectives de la quinoa, des pâtes et du riz dans le régime alimentaire des populations locales. Selon nous, les statistiques non référencées données par Jacobsen (2011: 396) pour affirmer que "la consommation de quinoa en Bolivie n'est que de $2 \mathrm{~kg}$ par personne et par an, alors qu'elle est de $25 \mathrm{~kg}$ pour le riz et les pâtes" sont donc dénuées de sens tant du point de vue nutritionnel que sur le plan socio-culturel. Nous concordons avec Jacobsen (2011: 396) sur le fait que la quinoa a été progressivement remplacée par les pâtes et le riz qui, contrairement à la quinoa de qualité ordinaire disponible sur les marchés ruraux et urbains de Bolivie, n'exigent ni tri ni lavage fastidieux avant d'être consommés. Mais ce changement s'est produit bien avant l'entrée de la quinoa sur le marché de l'exportation (voir Johnsson 1986: 167, se référant au début des années 80) et reflète la tendance générale observée dans les pays latino-américains depuis au moins 50 ans d'importer des céréales subventionnées venues d'Amérique du Nord (Hellin \& Higman 2005: 168). Il est donc incorrect de lier, comme le font Jacobsen (2011) et d'autres journalistes, le déclin de la consommation locale de quinoa à son succès récent sur le marché international. De fait, le jugement de Jacobsen ignore les efforts récemment déployés par le gouvernement bolivien pour promouvoir la consommation locale de quinoa en l'incluant dans plusieurs programmes de sécurité alimentaire comme "desnutrición cero", "desayuno escolar" et "subsidio de lactancia familiar" (MDRyT (Ministerio de Desarrollo Rural y Tierras) CONACOPROQ (Concejo Nacional de Comercializadores y Productores de Quinua),2009).

\section{Un diagnostic erroné et des solutions douteuses... pour menacer davantage la durabilité agricole ?}

Le diagnostic présenté par Jacobsen (2011) comporte de nombreuses erreurs et des simplifications abusives (p.ex., page 391 , la gamme des températures extrêmes allant de $-11{ }^{\circ} \mathrm{C}$ à $30^{\circ} \mathrm{C}$ combine une moyenne saisonnière des températures minimales et une valeur extrême journalière, ce qui est incohérent). Plus critique pour sa démonstration est l'affirmation selon laquelle : "La frontière agricole s'est étendue, les terres vierges des plaines ayant été labourées" (Jacobsen 2011: 392). II y a manifestement une confusion ici puisque les terres labourées dans les zones basses de l'altiplano sud ne sont pas des terres vierges mais des pâturages, utilisés depuis des temps reculés par les populations locales pour leurs élevages de lamas et de moutons. Cette expansion des cultures de 
quinoa au détriment de pâturages (non de terres vierges) résulte du choix délibéré des paysans locaux. Un problème réel, à savoir la conversion de pâturages collectifs en parcelles cultivées d'usage privatif, avec leurs corolaires de changements fonciers et de marginalisation de l'élevage, se trouve donc caricaturé par Jacobsen (2011) en un processus d'expansion d'une frontière agricole sur des terres vierges. Cette simplification permet alors à l'auteur d'écrire : "l'incorporation de terres vierges dans la zone de production contrevient aux standards élémentaires de l'IFOAM" (Jacobsen 2011: 393). À l'évidence, il s'agit là d'une conclusion abusive déformant la complexité socioenvironnementale du changement d'usage des terres agricoles dans la région.

Plusieurs solutions sont proposées par Jacobsen (2011: 394) pour traiter les divers problèmes agrotechniques qu'il a identifiés. Certaines de ces solutions semblent pour le moins douteuses et l'on se demande, par exemple, comment les aménagements de champs surélevés (suka kollos) pourraient être mis en place, comme le suggère Jacobsen (2011: 395), dans une région aussi aride que l'altiplano sud alors que cette technique pré-hispanique a été conçue pour les zones inondables proches du Lac Titicaca ou celles des plaines amazoniennes (Denevan 2001). Concernant l'irrigation, elle est employée traditionnellement dans l'altiplano sud, mais seulement à très petite échelle, à partir de rares sources d'eau superficielles. Cette irrigation traditionnelle est sans commune mesure avec la préconisation du projet CPTS (CTPS 2011) mentionné par Jacobsen (2011: 394) de forer 200 puits dans des aquifères fossiles pour irriguer 1 million d'hectares de quinoa dans l'altiplano sud de Bolivie (à comparer aux 52.000 ha environ actuellement cultivés sur l'ensemble du territoire national). Si un tel projet devait se réaliser, il est à espérer qu'avant de l'avaliser les agences financières évalueraient de façon scrupuleuse ses impacts potentiels sur la durabilité environnementale, sociale et économique dans la région.

L' irrigation déficitaire est souvent présentée comme une solution possible pour une production durable de la quinoa, avec l'argument qu'un rendement amélioré et plus stable dans les champs irrigués réduirait la nécessité d'étendre les surfaces cultivées pour augmenter la production totale de quinoa. Jacobsen (2011: 394-395) expose longuement les bénéfices potentiels de l'irrigation déficitaire, en particulier sous la forme de l'irrigation alternée (ARD) et de l'irrigation au goutte-àgoutte. Tout en signalant rapidement les risques potentiels de salinisation des sols ou d'épuisement des nappes phréatiques, Jacobsen (2011: 395) conclut que l'irrigation déficitaire représente "un grand potentiel pour accroître la production agricole et améliorer le niveau de vie dans les zones sèches". Se fondant sur des études menées au Proche-Orient et en Afrique du Nord (Oweis \& Hachum 2006), sa conclusion ignore les résultats du programme de recherche QUINAGUA sur l'irrigation déficitaire dans l'altiplano bolivien (Geerts et al. 2008a), pourtant brièvement cité en introduction. Dans le cas particulier de l'altiplano sud, avec des ressources en eau rares et souvent salines, Geerts et al. (2008b) avertissent que "l'irrigation déficitaire avec une eau de mauvaise qualité et sur des parcelles cultivées sur une nappe saline présente un danger sérieux pour une production durable de quinoa". Ces auteurs concluent que : "bien que potentiellement bénéfique, l'irrigation déficitaire de la quinoa dans des régions aussi arides que le sud de l'altiplano bolivien doit être considérée avec précaution". Incidemment, une autre question sans réponse serait de savoir comment des systèmes d'irrigation au goutte-à-goutte ou des procédures d'arrosages aussi sophistiquées que l'ARD pourraient fonctionner de manière fiable à $3.600 \mathrm{~m}$ d'altitude sous un rayonnement solaire extrême et des gelées nocturnes fréquentes même en été (Pouteau et al. 2011). On ne voit pas bien non plus comment, sans d'importants financements extérieurs (et avec la 
dépendance qui les accompagne...), des techniques aussi avancées pourraient être implantées et gérées de façon durable dans une des régions les plus déshéritées d'Amérique Latine.

\section{Conclusion}

L'essor actuel de la production de quinoa dans l'altiplano sud de Bolivie soulève des interrogations justifiées quant à la durabilité sociale et écologique dans la région (Reynolds et al. 2008, Winkel 2008, Winkel et al. 2011). Au vu des changements rapides du système de culture, menaçant potentiellement la base écologique d'une production durable de la quinoa, certains observateurs n'hésitent pas à prendre leurs " impressions personnelles" (Jacobsen 2011: 392) pour les preuves fiables d'un désastre environnemental et de menaces imminentes pour la sécurité alimentaire des populations locales. Laissant de côté les relations erronées établies entre des statistiques nationales de rendements en quinoa et la fertilité locale du sol, ainsi que les affirmations infondées concernant le régime alimentaire des paysans boliviens, nous ne disposons que d'une connaissance restreinte des bases agro-écologiques et sociales de la durabilité de la quinoa dans l'altiplano sud de Bolivie. Récemment, des programmes de recherche nationaux et internationaux ont commencé à examiner les questions complexes liées à la durabilité agricole dans cette région reculée (p.ex. ARIDnet, EQUECO, IFAD-NUS, PIEB-MDRT-MPD, QUINAGUA). Les résultats préliminaires de ces programmes montrent l'importance des aspects agro-écologiques, notamment ceux liés à la structure et à l'organisation des paysages (Winkel 2011). Mais à côté de préoccupations proprement écologiques, les conclusions de ces programmes soulignent le rôle fondamental de questions socio-économiques touchant le foncier et la conversion de pâturages collectifs en parcelles d'usage privatif, ou encore la complexité des combinaisons d'activités agricoles et non-agricoles gérées par les producteurs de quinoa à la recherche d'une vie meilleure pour eux et leurs enfants (Chaxel 2007, Vassas et al. 2008).

Nous estimons que la diffusion des arguments développés par Jacobsen (2011), même s'ils sont scientifiquement infondés (ou précisément à cause de cela), peut avoir un impact négatif sur les personnes œuvrant pour une production alimentaire durable et des relations commerciales équitables avec les petits producteurs des pays en développement. Alarmés à tort par les conséquences supposées négatives de leur choix de consommation, certains acteurs des pays du Nord pourraient renoncer à leur soutien aux producteurs de quinoa des Andes. En conclusion nous considérons que, plutôt que de contrôles agro-techniques renforcés sur ces paysans du Sud, la concurrence croissante sur le marché international de la quinoa a besoin d'ajustements de la part des pays les plus riches en vue d'introduire davantage d'éthique dans leurs relations économiques avec les pays exportateurs et dans la coopération avec les producteurs locaux. Le fondement de ces relations éthiques en économie et en recherche réside dans une participation active de leurs bénéficiaires ultimes, à savoir les producteurs locaux. Ceci implique un centrage sur les besoins et les réalités propres de ces acteurs, et leur association continue au processus complet de définition des problèmes, d'élaboration des connaissances et de prise de décisions (ISE 2006). En ce qui concerne la gestion et la recherche agricoles, cette approche éviterait ce qu'Holling \& Meffe (1996) qualifient de pathologie de la gestion des ressources naturelles par commandement et control (command and control) (voir aussi Stallman 2011), et ouvrirait une perspective davantage fondée sur la gestion collective des ressources naturelles (Ostrom 2010) et le droit à une alimentation adéquate (De Schutter 2011). 


\section{Remerciements}

Les auteurs remercient plusieurs chercheurs boliviens et les correcteurs anonymes pour leurs commentaires sur le manuscrit. Les co-auteurs français ont bénéficié de l'appui financier de l'ANR (Agence Nationale de la Recherche) dans le cadre du programme "Agriculture et Développement Durable", projet "ANR-06-PADD-011-EQUECO".

\section{Références}

Acosta-Alba, l., 2007: Durabilité des systèmes de production de l'altiplano sud bolivien : quels équilibres entre élevage et agriculture ? Mémoire de Master "Sciences et Technologies du Vivant". AgroParisTech, Université Paris 7. Paris, France. 44 p. + annexes. Disponible à : $\underline{w w w . i r d . f r / e q u e c o}$

APSA II, 2008: Gestión del componente de investigación agropecuaria. Programa de apoyo programatico al sector agropecuario, APSA fase II, 2009-2010. DANIDA, Ministry de Foreign Affairs,Copenhagen, Denmark, 56 pp.

Astudillo, D., 2007: The role de quinoa in the diets and livelihoods de households in the Southern Bolivian Altiplano: a case study in the municipalities de Salinas and Colcha K. Biodiversity International, Congressional Hunger Center. Rome, Italy. Disponible à : http://www.underutilized-species.org/record details.asp?id=824

Chaxel, S., 2007: Trajectoires de vie des familles de la zone Intersalar (Bolivie) et changements de pratiques agricoles. Mémoire de Master "Ingéniérie en Agronomie Tropicale' IRC-SupAgro. Montpellier, France. 90 p. + annexes. Disponible à : $\underline{w w w . i r d . f r / e q u e c o}$

Cossio, J., 2008: Agricultura de conservación con un enfoque de manejo sostenible en el Altiplano sur. Revista Hábitat 75, 44-49. Disponible à : http://www.lidema.org.bo/

CTPS (Centro de Promoción de Tecnologías Sostenibles), 2011: Desarrollo agrícola sostenible de la quinua en tierras áridas, no tradicionales, del Altiplano boliviano. Taller "Avances en la sostenibilidad de la quinua en Bolivia". CABOLQUI. Mayo de 2011. La Paz, Bolivia.

Denevan, W.M., 2001: Cultivated landscapes de native Amazonia and the Andes. Oxford University Press. New York, USA.

De Schutter, O., 2011: The right of everyone to enjoy the benefits of scientific progress and the right to food: from conflict to complementarity. Human Rights Quarterly 33, 304-350.

Félix, D., 2008: Cultivo sostenible de la quinua en Bolivia: prácticas individuales y reglas comunitarias. Report de Project "Sostenibilidad de los sistemas de producción de las familias indígenas de los municipios de Llica, Tahua y Salinas de Garci Mendoza". Agrónomos y Veterinarios sin Fronteras, 26 p.

Geerts, S., D. Raes, M. Garcia, J. Vacher, R. Mamani, J. Mendoza, R. Huanca, B. Morales, R. Miranda, J. Cusicanqui, C. Taboada, 2008a: Introducing deficit irrigation to stabilize yields of quinoa (Chenopodium quinoa Willd.). European Journal of Agronomy 28, 427-436.

Geerts, S., D. Raes, M. Garcia, O. Condori, J. Mamani, R. Miranda, J. Cusicanqui, C. Taboada, E. Yucra, J. Vacher, 2008b: Could deficit irrigation be a sustainable practice for quinoa (Chenopodium quinoa Willd.) in the Southern Bolivian Altiplano? Agricultural Water Management 95, 909-917. 
Hellin, J., S. Higman, 2005: Crop diversity and livelihood security in the Andes. Development in Practice 15, 165174.

Héran, V., 2011: L'Altiplano Sud de Bolivie dans la mondialisation agricole : quelles solutions pour une production durable de quinoa ? Cas des communautés du municipio de Tomave, département de Potosí. MSc Thesis. ISTOM, Cergy-Pontoise, France. Disponible à : $\underline{\text { www.ird.fr/equeco }}$

Holling, C.S., G.K. Meffe, 1996: Command and control and the pathology of natural resource management. Conservation Biology 10, 328-333.

ISE - International Society of Ethnobiology, 2006: International Society of Ethnobiology Code of Ethics (with2008 additions). Disponible à : http://ethnobiology.net/code-of-ethics/

Jacobsen, S.E., 2011. The situation for quinoa and its production in southern Bolivia: from economic success to environnemental disaster. Journal of Agronomy and Crop Science 197, 390-399.

Johnsson, M., 1986: Food and culture among Bolivian Aymara: symbolic expressions de social relations. Acta Universitatis Upsaliensis, Uppsala Studies in Cultural Anthropology 7. Uppsala, Sweden. 188 p.

Laguna, P., 2008: Grano pequeño, mercado pequeño, grandes apuestas: estudiando los limites de la régulacion estatal francesa del comercio justo a partir del caso de la quinua. FTIS 2008 : 3me Colloque International sur le Commerce Equitable. Montpellier, France.

MDRyT (Ministerio de Desarrollo Rural y Tierras) - CONACOPROQ (Concejo Nacional de Comercializadores y Productores de Quinua) , 2009: Política Nacional de la Quinua. La Paz, Bolivia. 118 p.

Montoya Choque, J.C., 2007: Estimación del consumo de la quinua en la ciudad de Oruro. Fundación AUTAPO. Oruro, Bolivia. 84 p. + anexos.

National Research Council, 1989: Lost crops de the Incas: little-known plants de the Andes with promise for worldwide cultivation. National Academy Press. Washington, D.C., USA.

Ofstehage, A., 2010: The gift de the middleman: an ethnography de quinoa trading networks in Los Lipez de Bolivia. MSc Thesis. Department de Social Sciences, Wageningen University, Wageningen, The Netherlands. 133 p. Disponible à : http://ssrn.com/abstract $=1866330$

Ostrom, E., 2010. La gouvernance des biens communs : pour une nouvelle approche des ressources naturelles. Editions De Boeck. Bruxelles, Belgique. 301 p.

Oweis, T., A. Hachum, 2006: Water harvesting and supplemental irrigation for improved water productivity de dry farming systems in West Asia and North Africa. Agricultural Water Management 80, 57-73.

PIEB (Programa de Investigación Estratégica en Bolivia), 2009: Convocatoria: Formulación de propuestas para la producción sostenible de quinua en Oruro y Potosí. Ministerio de Planificación del Desarrollo, Ministerio de Desarrollo Rural y Tierras, 43 pp. La Paz, Bolivia.

Pouteau, R., S. Rambal, J.P. Ratte, F. Gogé, R. Joffre, T. Winkel, 2011: Downscaling MODIS-derived maps using GIS and boosted regression trees: the case de frost occurrence over the arid Andean highlands of Bolivia. Remote Sensing of Environnement 115, 117-129.

Reynolds J.F., E. Huber-Sannwald, J.E. Herrick, 2008a: La sustentabilidad de la producción de la quinua en el Altiplano sur de Bolivia: aplicación del paradigma de desarrollo de zonas secas. Revista Hábitat 75, 10-15. Disponible à : http://www.lidema.org.bo/ 
Reynolds J.F., E. Huber-Sannwald, J.E. Herrick, 2008b: Un nuevo paradigma para el rendimiento y el combate de la desertificación. Revista Hábitat 75, 4-7. Disponible à : http://www.lidema.org.bo/

Reynolds, J.F., D.M. Stafford Smith, E.F. Lambin, B.L. Turner II, M. Mortimore, S.P.J. Batterbury, T.E. Downing, H. Dowlatabadi, R.J. Fernández, J.E. Herrick, E. Huber-Sannwald, H. Jiang, R. Leemans, T. Lynam, F.T. Maestre, M. Ayarza, B. Walker, 2007: Global desertification: building a science for dryland development. Science 316, 847851.

Rojas W., J.L. Soto, E. Carrasco, 2004: Study on the social, environnemental and economic impacts of quinoa

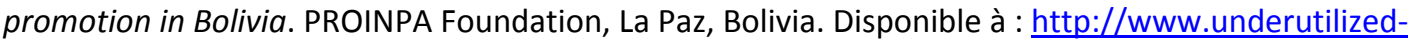
species.org/Documents/PUBLICATIONS/quinoa case study en.pdf

Stallman, H.R., 2011: Ecosystem services in agriculture: determining suitability for provision by collective management. Ecological Economics 71, 131-139.

Tapia M.E., C. Morón, G. Ayala, A.M. Fries, 2000: Valor nutritivo y patrones de consumo. In: Tapia ME, ed. Cultivos andinos subexplotados y su aporte a la alimentación. FAO, Santiago, Chile. Disponible à : http://www.rlc.fao.org/es/agricultura/produ/cdrom/contenido/libro10/home10.htm

Vassas, A., M. Vieira Pak, J.R. Duprat, 2008: El auge de la quinua: cambios y perspectivas desde una visión social. Revista Hábitat 75, 31-35. Disponible à : http://www.lidema.org.bo/

Winkel, T., 2008: El éxito de la quinua en el comercio mundial: una oportunidad para investigar la sostenibilidad socio ecológica en el Altiplano sur de Bolivia. Revista Hábitat 75, 25-28. Disponible à :

http://www.lidema.org.bo/

Winkel, T. (coord.), 2011: Pour durer, changeons : paradoxes et leçons du succès de la quinoa. Rapport scientifique final du projet EQUECO - Émergence de la quinoa dans le commerce mondial : quelles conséquences sur la durabilité sociale et agricole dans l'Altiplano Bolivien ? . Cooperation Franco-Bolivienne. ANR (Agence Nationale de la Recherche), Projet ANR-06-PADD-011. CIRAD/CNRS/EHESS/INRA/IRD (coord.)/UM3. Montpellier, France. 83 p. Disponible à : www.ird.fr/equeco 